\title{
Contamination in the Rare-Earth Element Orthophosphate Reference Samples
}

\section{John J. Donovan}

Department of Geological Sciences,

The University of Oregon,

Eugene, OR 97403-1272

\section{John M. Hanchar}

Department of Earth and Environmental

Sciences,

The George Washington University,

Washington, DC 20006

\section{Phillip M. Picolli}

Department of Geology,

The University of Maryland,

College Park, MD 20742

\section{Marc D. Schrier}

Department of Chemistry,

The University of California,

Berkeley, CA 94720

\section{Lynn A. Boatner}

Solid State Division, Oak Ridge

National Laboratory,

Oak Ridge, TN 37831

\section{and}

Eugene Jarosewich

Department of Mineral Sciences,

Smithsonian Institution,

Washington, DC 20560
Several of the fourteen rare-earth element (plus Sc and Y) orthophosphate standards grown at Oak Ridge National Laboratory in the 1980s and widely distributed by the Smithsonian Institution's Department of Mineral Sciences, are significantly contaminated by $\mathrm{Pb}$. The origin of this impurity is the $\mathrm{Pb}_{2} \mathrm{P}_{2} \mathrm{O}_{7}$ flux that is derived from the thermal decomposition of $\mathrm{PbHPO}_{4}$. The lead pyrophosphate flux is used to dissolve the oxide starting materials at elevated temperatures $\left(\approx 1360^{\circ} \mathrm{C}\right)$ prior to the crystal synthesis. Because these rare-earth element standards are extremely stable under the electron beam and considered homogenous, they have been of enormous value to electron probe micro- analysis (EPMA). The monoclinic, monazite structure, orthophosphates show a higher degree of $\mathrm{Pb}$ incorporation than the tetragonal xenotime structure, orthophosphates. This paper will attempt to describe and rationalize the extent of the $\mathrm{Pb}$ contamination in these otherwise excellent materials.

Key words: EPMA; microanalysis; orthophosphates; quantitative analysis; rare earth elements; rare earth phosphates; REE; standards.

Accepted: August 22, 2002

Available online: http://www.nist.gov/jres

\section{Introduction}

Highly accurate analyses from the electron microprobe analyzer (EMPA) are only (but not solely) obtainable through the use of well-characterized and stable standards containing a major and/or known concentration of the element in question. For the rare earth elements (REE) this goal has, until recently, been elusive due to the lack of specimens exhibiting these vital properties.

The lanthanide orthophosphates, consisting of compounds with the stoichiometry $\mathrm{LnPO}_{4}$ where Ln represents any of the REE in the series extending from La to $\mathrm{Lu}$ (plus the related compounds $\mathrm{YPO}_{4}$ and $\mathrm{ScPO}_{4}$ ), are 
chemically durable and radiation resistant refractory materials. During the early 1980s a variety of single crystal rare earth orthophosphate samples were synthesized at Oak Ridge National Laboratory and the structures determined from x-ray refinements $[1,2,3,4,5$, and 6]. The primary purposes of these studies were varied, but they included nuclear and actinide waste disposal and scintillator material research as well as fundamental materials characterization investigations. The crystals were synthesized using a high-temperature solvent (flux-growth) technique, the details of which are available from the original papers, and a good overview of the development of these orthophosphates is discussed in Boatner and Sales [7], and references therein.

One interesting fact is that although the starting materials were carefully selected to be free from REE impurities, they were grown in a lead pyrophosphate $\left(\mathrm{PbHPO}_{4}\right)$ flux. $\mathrm{Pb}$ contamination was not a concern for the original purposes of those experiments, however its presence was detected early on, and the solid state chemistry (but not the concentration) of $\mathrm{Pb}$ in the orthophosphate was characterized by means of electron paramagnetic resonance spectroscopy (EPR) [8]. Subsequently, these materials were investigated for possible use as standards for EPMA by the Smithsonian Institution [9], and put through a series of tests. These included homogeneity testing and a comparison to the commonly used REE doped aluminum silicate glass standards of Drake and Weill [10] using the EPMA, and a check of 10 selected REE contaminants on 7 of the compounds using instrumental neutron activation analysis. The materials appeared to be robust under electron bombardment, did not oxidize or seem hygroscopic, and no serious contamination or inhomogeneities were noted at the time and these efforts were followed by a general distribution of the material to interested parties.

In the late 1990s it was reported to one of us (JJD) that at least one investigator (E. J. Essene, University of Michigan, personal communication) had raised the issue of the role of the $\mathrm{Pb}$ impurity in some of the REE phosphate standards. The $\mathrm{Pb}$ impurity is especially significant in the $\mathrm{CePO}_{4}$ crystals whose black coloration is consistent with possible mixed valence $\left(\mathrm{Ce}^{3+}-\mathrm{Ce}^{4+}\right)$ effects-the presence of which could alter the high-temperature solid-state chemical properties and lead to an enhanced incorporation of $\mathrm{Pb}$ during the crystal-growth process. Subsequent investigations of the materials revealed $\mathrm{Pb}$ ranging in concentration from less than 0.01 mass fraction to more than 0.04 mass fraction in the $\mathrm{CePO}_{4}$, depending on the specific grains analyzed. It is the intent of this paper to characterize the extent of the $\mathrm{Pb}$ contamination in these otherwise extremely useful standards for EPMA.

\section{Experimental Methods}

Quantitative wavelength dispersive spectrometry (WDS) analyses for the REEs Sc, Y, and $\mathrm{Pb}$ in each of the 16 orthophosphate samples were done using a Cameca SX-5 $1^{1}$ electron microprobe at $20 \mathrm{keV}, 20 \mathrm{nA}$ $\left(2.0 \times 10^{-8} \mathrm{~A}\right)$, using a $10 \mu \mathrm{m}$ beam diameter at UC Berkeley. In addition, one of the Drake and Weill REE glasses [10], and two other REE doped calcium aluminum silicate discussed in Roeder [11] and Roeder et al. [12] were analyzed. For quantitative analyses, the $\mathrm{K}_{\alpha}$ $\mathrm{x}$-ray line was used for $\mathrm{Sc}, \mathrm{L}_{\alpha}$ lines for $\mathrm{Y}$ and the other REE elements, and the $\mathrm{M}_{\alpha}$ line was used for $\mathrm{Pb}$. Count times were $20 \mathrm{~s}$ on peak and $10 \mathrm{~s}$ on each off-peak position except for $\mathrm{Pb}$ where the count times were doubled, respectively.

A complete description of the analytical setup and secondary standard accuracy for the analyzed elements (the composition of the REE phosphate primary standards in these cases had been previously adjusted for average $\mathrm{Pb}$ concentrations) is presented in Table 1 . Secondary standards included synthetic yttrium-aluminum garnet (YAG) and alamosite $\left(\mathrm{PbSiO}_{3}\right)$ from Tsumeb, Namibia and were assumed to be stoichiometric for Y and $\mathrm{Pb}$, respectively. The Roeder REE glass S-254 [12] was assumed to have a nominal concentration $\left(1.04 \times 10^{-2}\right.$ mass fraction) for $\mathrm{La}, \mathrm{Ce}, \mathrm{Pr}, \mathrm{Nd}, \mathrm{Sm}, \mathrm{Dy}$, $\mathrm{Ho}, \mathrm{Er}, \mathrm{Yb}$, and $\mathrm{Lu}$, and the Drake and Weill REE-1 glass was used based on published concentrations for $\mathrm{Eu}, \mathrm{Gd}, \mathrm{Tb}$, and $\mathrm{Tm}[10]$. For all rare-earth elements, the relative differences obtained when comparing the secondary standards to the primary standard is better than $10 \%$ at the 0.01 mass fraction to 0.04 mass fraction concentration levels and better than $6 \%$ in all but three cases (Pr, Sm and Lu).

The difficulty of dealing with interfering elements for REE analyses using the $\mathrm{L}_{\alpha} \mathrm{X}$-ray lines is painfully evident in even cursory WDS spectral scans on these samples and can only be overcome by careful and consistent application of an automatic correction scheme. Table 2 shows the REEs that interfere with the analyzed elements. These were interferences quantitatively corrected for using the iteration method of Donovan et al. [13], that is especially well suited for using large magnitude interferences for trace element determinations. For the $\mathrm{Pb}$ analyses, the $\mathrm{M}_{\alpha}$ line was used with a quantitative interference correction for $\mathrm{Y}$ (possible high order interferences from $\mathrm{La}$ and $\mathrm{Tb}$ were not observed). Standard

\footnotetext{
${ }^{1}$ NIST disclaimer: Certain commercial equipment, instruments, or materials are identified in this paper to foster understanding. Such identification does not imply recommendation or endorsement by the National Institute of Standards and Technology, nor does it imply that the materials or equipment identified are necessarily the best available for the purpose.
} 
Table 1. Analytical setup and measured differences between the secondary standards and the primary standard for REE quantitative analysis ${ }^{\mathrm{a}}$

\begin{tabular}{|c|c|c|c|c|}
\hline $\begin{array}{l}\text { Element } \\
\text { Sc } \mathbf{K}_{\alpha}\end{array}$ & $\begin{array}{l}\text { Spect. setup } \\
\text { LiF (FPC-2) }\end{array}$ & $\begin{array}{l}\text { Primary standard } \\
\mathrm{ScPO}_{4} \text { (syn.) }\end{array}$ & $\begin{array}{c}\text { Secondary standard } \\
\text { Conc in mass fraction } \times 10^{2}\end{array}$ & Relative diff. \\
\hline $\mathrm{YL}_{\alpha}$ & PET (FPC-1) & $\mathrm{YPO}_{4}$ (syn.) & YAG (stoic.) & $+0.368,+0.82 \%$ \\
\hline $\mathrm{LaL}_{\alpha}$ & $\mathrm{LiF}(\mathrm{FPC}-2)$ & $\mathrm{LaPO}_{4}$ (syn.) & S-254 (1.04 nom.) & $-0.020,-1.92 \%$ \\
\hline $\mathrm{CeL}_{\alpha}$ & LiF (FPC-2) & $\mathrm{CePO}_{4}$ (syn.) & S-254 (1.04 nom.) & $-0.010,-0.95 \%$ \\
\hline $\operatorname{PrL}_{\alpha}$ & LiF (FPC-2) & $\mathrm{PrPO}_{4}$ (syn.) & S-254 (1.04 nom.) & $-0.103,-9.95 \%$ \\
\hline $\mathrm{NdL}_{\alpha}$ & LiF (FPC-2) & $\mathrm{NdPO}_{4}$ (syn.) & S-254 (1.04 nom.) & $-0.007,-0.70 \%$ \\
\hline $\mathrm{SmL}_{\alpha}$ & LiF (FPC-2) & $\mathrm{SmPO}_{4}$ (syn.) & S-254 (1.04 nom.) & $-0.055,-5.27 \%$ \\
\hline $\mathrm{EuL}_{\alpha}$ & $\mathrm{LiF}($ FPC-2) & $\mathrm{EuPO}_{4}$ (syn.) & REE-1 (3.63 pub.) & $+0.069,+1.90 \%$ \\
\hline $\mathrm{GdL}_{\alpha}$ & LiF (FPC-2) & $\mathrm{GdPO}_{4}$ (syn.) & REE-1 (3.87 pub.) & $-0.012,-0.31 \%$ \\
\hline $\mathrm{TbL}_{\alpha}$ & LiF (FPC-2) & $\mathrm{TbPO}_{4}$ (syn.) & REE-1 (3.78 pub.) & $-0.116,-3.08 \%$ \\
\hline $\operatorname{DyL}_{\alpha}$ & $\mathrm{LiF}(\mathrm{FPC}-2)$ & $\mathrm{DyPO}_{4}$ (syn.) & S-254 (1.04 nom.) & $-0.035,-3.35 \%$ \\
\hline $\mathrm{HoL}_{\alpha}$ & $\mathrm{LiF}(\mathrm{FPC}-2)$ & $\mathrm{HoPO}_{4}$ (syn.) & S-254 (1.04 nom.) & $-0.041,-3.92 \%$ \\
\hline $\operatorname{ErL}_{\alpha}$ & $\mathrm{LiF}(\mathrm{FPC}-2)$ & $\mathrm{ErPO}_{4}$ (syn.) & S-254 (1.04 nom.) & $-0.047,-4.51 \%$ \\
\hline $\operatorname{TmL}_{\alpha}$ & $\mathrm{LiF}(\mathrm{FPC}-2)$ & $\mathrm{TmPO}_{4}$ (syn.) & REE-1 (3.81 pub.) & $-0.127,-3.33 \%$ \\
\hline $\mathrm{YbL}_{\alpha}$ & LiF (FPC-2) & $\mathrm{YbPO}_{4}$ (syn.) & S-254 (1.04 nom.) & $-0.047,-4.53 \%$ \\
\hline $\mathrm{LuL}_{\alpha}$ & LiF (FPC-2) & $\mathrm{LuPO}_{4}$ (syn.) & S-254 (1.04 nom.) & $-0.103,-9.94 \%$ \\
\hline $\mathrm{PbM}_{\alpha}$ & PET (FPC-1) & $\mathrm{PbCO}_{3}$ (Tsumeb) & $\mathrm{PbSiO}_{3}$ (stoic.) & $+0.550,+0.75 \%$ \\
\hline
\end{tabular}

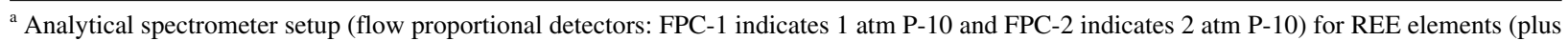
$\mathrm{Sc}, \mathrm{Y}$, and $\mathrm{Pb}$ ) and results of secondary standard measurements (algebraic difference and relative difference) performed at UC Berkeley. All elements were measured at $20 \mathrm{keV}, 20 \mathrm{nA}(150 \mathrm{nA}$ for the four grain map), $10 \mu \mathrm{m}$ beam diameter, $20 \mathrm{~s}$ on-peak integration time and $10 \mathrm{~s}$ on each off-peak except for $\mathrm{Pb}$ which was counted for $40 \mathrm{~s}$ on-peak and $20 \mathrm{~s}$ on each off-peak position $(240 \mathrm{~s}$ on-peak and $120 \mathrm{~s}$ on each off-peak position for the four grain map in Fig. 5). Each result shown is the average of 10 measurements.

and background intensities along with the calculated $P / B$ (peak to background) for each line in its associated primary standard are shown in Table 3.

Under the analytical conditions which were utilized at Berkeley, the minimum detection limits for both single analyses calculated from Love and Scott [14], and for the average of 10 replicate analyses based on Goldstein et al. [15], are shown in Table 4. Minimum detection limits for 10 replicate analyses based on the actual measured standard deviation are about $3.0 \times 10^{-4}$ mass fraction to $6.0 \times 10^{-4}$ mass fraction for all elements in all matrices although only values for $\mathrm{CePO}_{4}$ or $\mathrm{GdPO}_{4}$ are shown in Table 4. A measured detection limit of $3 \times 10^{-4}$ mass fraction to $6 \times 10^{-4}$ mass fraction for the average of 10 replicates at $99 \%$ a confidence level was typical for the REE analyses under these conditions. The $\mathrm{Pb}$ detection limit at a $99 \%$ confidence level was about $4.5 \times 10^{-4}$ mass fraction.

Another set of measurements, for the analysis of $\mathrm{Pb}$ homogeneity only, were also done on the same grains, but in a different area from the REE and $\mathrm{Pb}$ measurements done at UC Berkeley. These measurements were made for each REE orthophosphate using a JEOL 8900 Superprobe at the University of Maryland-College Park. $\mathrm{X}$-ray intensities of $\mathrm{Pb}$ were obtained using an accelerating voltage of $20 \mathrm{keV}$, and a beam current of $150 \mathrm{nA}$. Count times were $60 \mathrm{~s}$ on peak, and $30 \mathrm{~s}$ for backgrounds on each side of the peak. $\mathrm{Pb}$ was analyzed using a PETH (which utilizes a smaller diameter Rowland Circle allowing for higher count rates, but has poorer wavelength resolution) crystal, and background positions of $+4 \mathrm{~mm}(L=173.307 \mathrm{~mm}$ or $5.4013 \AA)$ and $-3 \mathrm{~mm}$ $(L=166.307 \mathrm{~mm}$ or $5.1828 \AA)$. Natural cerussite $\left(\mathrm{PbCO}_{3}\right)$ from Tsumeb, Namibia, was used as a standard for $\mathrm{Pb}(0.8393$ mass fraction $\mathrm{PbO})$. It should be noted that although cerussite is a carbonate mineral it did not appear to degrade under the electron beam during the analyses. The $\mathrm{Pb} \mathrm{M} \mathrm{M}_{\alpha} \mathrm{x}$-ray line was used for all analyses, with the exception of $\mathrm{YPO}_{4}$, where $\mathrm{M}_{\beta}$ was used due to an interference from $\mathrm{Y}_{1 \gamma 3}$ on $\mathrm{Pb} \mathrm{M}_{\alpha}$. For these $\mathrm{Pb}$ homogeneity measurements, the REE and phosphate concentrations were not measured but were incorporated as stoichiometric proportions into the ZAF algorithm in order to approximately account for matrix effects. The single analysis detection limit at a $99 \%$ confidence level for $\mathrm{Pb}$ under these analytical conditions was about $1.4 \times 10^{-4}$ mass fraction $\mathrm{Pb}$ based on a standard count rate of $263.9 \mathrm{cps} / \mathrm{nA}$ and a background of $0.8 \mathrm{cps} / \mathrm{nA}$ measured on $\mathrm{CePO}_{4}$.

Measurements were done on two different sets of REE orthophosphate samples. The first set consists of material for 16 orthophosphates, including Sc and Y obtained from one of us (JMH) and mounted along with primary and secondary standards for analysis and interference corrections. These materials were mounted in a $25 \mathrm{~mm}$ diameter acrylic mount approximately $1.5 \mathrm{~cm}$ deep using a cold set epoxy and circulated to both laboratories. This sample will be referred to as the "Round Robin" mount in the discussion that follows. 
Table 2. Quantitative interferences. ${ }^{a}$ Also listed are the wavelengths (in $\AA$ ) of the $\mathrm{x}$-ray lines

\begin{tabular}{|c|c|}
\hline$\underset{\AA}{\text { Element }}$ & $\begin{array}{c}\text { On peak interferences } \\
\AA\end{array}$ \\
\hline $\mathrm{ScK}_{\alpha}$ at 3.0320 & $\operatorname{ErL}_{\beta 2}$ (II) at 3.0284 \\
\hline $\mathrm{YL}_{\alpha}$ at 2.6657 & $\mathrm{LaL}_{\gamma 1}$ (III)at 6.4260 (not observed) \\
\hline $\mathrm{LaL}_{\alpha}$ at 2.6657 & $\mathrm{NdLl}(\mathrm{I})$ at 2.6766 \\
\hline \multicolumn{2}{|l|}{$\mathrm{CeL}_{\alpha}$ at 2.5615} \\
\hline \multirow[t]{2}{*}{$\operatorname{PrL}_{\alpha}$ at 2.4630} & $\mathrm{LaL}_{\beta 1,4}(\mathrm{I})$ at $2.4595,2.4595$ \\
\hline & $\mathrm{SmLl}(\mathrm{I})$ at 2.4826 \\
\hline \multirow[t]{2}{*}{$\mathrm{NdL}_{\alpha}$ at 2.3704} & $\mathrm{CeL}_{\beta 1,4}(\mathrm{I})$ at $2.3566,2.3499$ \\
\hline & $\mathrm{PbL}_{\alpha 1,2}$ (II) at $2.3504,2.3732$ \\
\hline \multirow[t]{2}{*}{$\mathrm{SmL}_{\alpha}$ at 2.1998} & $\mathrm{CeL}_{\beta 2}(\mathrm{I})$ at 2.2092 \\
\hline & $\operatorname{PrL}_{\beta 3}$ (I) at 2.2175 (not observed) \\
\hline \multirow[t]{2}{*}{$\mathrm{EuL}_{\alpha}$ at 2.1209} & $\mathrm{NdL}_{\beta 3}$ (I) at 2.1273 \\
\hline & $\operatorname{PrL}_{\beta 2}(\mathrm{I})$ at 2.1197 \\
\hline \multirow[t]{3}{*}{$\mathrm{GdL}_{\alpha}$ at 2.0468} & $\mathrm{CeL}_{\gamma 1}$ (I) at 2.0489 \\
\hline & $\mathrm{LaL}_{\gamma 2,3}$ (I) at $2.0462,2.0415$ \\
\hline & $\mathrm{NdL}_{\beta 2}(\mathrm{I})$ at 2.0365 \\
\hline \multirow[t]{4}{*}{$\mathrm{TbL}_{\alpha}$ at 1.9765} & $\mathrm{LaL}_{\gamma 4}(\mathrm{I})$ at 1.9834 \\
\hline & $\operatorname{PrL}_{\gamma 1}$ (I) at 1.9614 (not observed) \\
\hline & $\mathrm{SmL}_{\beta 3}$ (I) at 1.9627 (not observed) \\
\hline & $\mathrm{PbL}_{\beta 1,2}$ (II) at $1.9660,1.9650$ (not observed) \\
\hline \multirow[t]{2}{*}{$\mathrm{DyL}_{\alpha}$ at 1.9088} & $\mathrm{EuL}_{\beta 1,4}(\mathrm{I})$ at $1.9207,1.9258$ \\
\hline & YbLl (I) at 1.8946 (possibly observed) \\
\hline \multirow[t]{2}{*}{$\mathrm{HoL}_{\alpha}$ at 1.8450} & $\mathrm{GdL}_{\beta 1,4}(\mathrm{I})$ at $1.8472,1.8543$ \\
\hline & LuLl (I) at 1.8362 (not observed) \\
\hline \multirow[t]{2}{*}{$\mathrm{ErL}_{\alpha}$ at 1.7842} & $\mathrm{TbL}_{\beta 1,4}(\mathrm{I})$ at $1.7770,1.7867$ \\
\hline & $\mathrm{NdL}_{\gamma 2,3}(\mathrm{I})$ at $1.8015,1.7968$ \\
\hline \multirow[t]{3}{*}{$\mathrm{TmL}_{\alpha}$ at 1.7268} & $\operatorname{DyL}_{\beta 1,4}(\mathrm{I})$ at $1.7110,1.7212$ \\
\hline & $\mathrm{GdL}_{\beta 2}(\mathrm{I})$ at 1.7457 \\
\hline & $\mathrm{SmL}_{\gamma 1}$ (I) at 1.7275 \\
\hline \multirow[t]{5}{*}{$\mathrm{YbL}_{\alpha}$ at 1.6718} & $\operatorname{EuL}_{\gamma 1}(\mathrm{I})$ at 1.6577 \\
\hline & $\mathrm{SmL}_{\gamma 2}(\mathrm{I})$ at 1.6608 \\
\hline & $\mathrm{TbL}_{\beta 2}(\mathrm{I})$ at 1.6834 \\
\hline & $\mathrm{YK}_{\alpha 1}$ (II) at 1.6580 (possibly observed) \\
\hline & $\mathrm{HoL}_{\beta 4}(\mathrm{I})$ at 1.6597 (not observed) \\
\hline \multirow[t]{3}{*}{$\mathrm{LuL}_{\alpha}$ at 1.6195} & $\mathrm{HoL}_{\beta 3}(\mathrm{I})$ at 1.6207 \\
\hline & $\operatorname{DyL}_{\beta 2}(\mathrm{I})$ at 1.6241 \\
\hline & $\mathrm{GdL}_{\gamma 1}$ (I) at 1.5928 (possibly observed) \\
\hline \multirow[t]{3}{*}{$\mathrm{PbM}_{\alpha}$ at 5.2860} & $\mathrm{YL}_{\gamma 3}$ (I) at 5.2848 \\
\hline & $\mathrm{LaL}_{\alpha 1}$ (II) at 5.3326 (not observed) \\
\hline & $\mathrm{TbL}_{\beta 1}$ (III) at 5.3310 (not observed) \\
\hline
\end{tabular}

\footnotetext{
${ }^{a}$ Analyzed elements and interfering elements were quantitatively corrected by using the iteration method of Donovan et al. [12]. Many of these interferences are 1st order interferences and therefore are the same energy as the interfering line, and hence, cannot be reduced by the use of pulse height analysis (PHA). Selection of alternative (beta) lines is sometimes possible, but the resulting reduction in intensity will also reduce sensitivity.
}

The "Round Robin" mount was carefully analyzed for $\mathrm{Pb}$ at both Berkeley and College Park to check for interlaboratory differences since the analytical results of trace element measurements are extremely sensitive to differences in spectrometer resolution and placement of off-peak intensity measurement positions. Homogeneity measurements were also done on this mount at College Park to check for possible $\mathrm{Pb}$ variations within this material itself.
Additional $\mathrm{Pb}$ measurements were performed at UC Berkeley on other material that was originally resident in the laboratory standard collection to check for possible inter-batch differences in $\mathrm{Pb}$ contamination some of the material had been produced in several runs at Oak Ridge under possibly different growth conditions. Analyses on this material will be referred to as the "Berkeley" REE mount. 
Table 3. Standard peak and background intensities (linear interpolation method) ${ }^{\mathrm{a}}$

\begin{tabular}{|c|c|c|c|}
\hline Element & $\begin{array}{l}\text { Peak intensity } \\
(\mathrm{cps} / \mathrm{nA})\end{array}$ & $\begin{array}{l}\text { Background intensity } \\
\qquad(\mathrm{cps} / \mathrm{nA})\end{array}$ & Peak/Background \\
\hline $\mathrm{ScK}_{\alpha}$ & $49.3\left(\mathrm{ScPO}_{4}\right)$ & 0.2 & 246.5 \\
\hline $\mathrm{YL}_{\alpha}$ & $68.3\left(\mathrm{YPO}_{4}\right)$ & 0.5 & 136.6 \\
\hline $\mathrm{LaL}_{\alpha}$ & $38.5\left(\mathrm{LaPO}_{4}\right)$ & 0.3 & 128.3 \\
\hline $\mathrm{CeL}_{\alpha}$ & $45.4\left(\mathrm{CePO}_{4}\right)$ & 0.5 & 90.8 \\
\hline $\operatorname{PrL}_{\alpha}$ & $55.1\left(\mathrm{PrPO}_{4}\right)$ & 0.6 & 91.8 \\
\hline $\mathrm{NdL}_{\alpha}$ & $64.9\left(\mathrm{NdPO}_{4}\right)$ & 0.6 & 108.1 \\
\hline $\mathrm{SmL}_{\alpha}$ & $80.8\left(\mathrm{SmPO}_{4}\right)$ & 1.3 & 62.2 \\
\hline $\mathrm{EuL}_{\alpha}$ & $89.6\left(\mathrm{EuPO}_{4}\right)$ & 1.1 & 81.5 \\
\hline $\mathrm{GdL}_{\alpha}$ & $95.2\left(\mathrm{GdPO}_{4}\right)$ & 1.2 & 79.3 \\
\hline $\mathrm{TbL}_{\alpha}$ & $101.9\left(\mathrm{TbPO}_{4}\right)$ & 1.3 & 78.4 \\
\hline $\operatorname{DyL}_{\alpha}$ & $107.8\left(\mathrm{DyPO}_{4}\right)$ & 1.5 & 71.9 \\
\hline $\mathrm{HoL}_{\alpha}$ & $113.6\left(\mathrm{HoPO}_{4}\right)$ & 2.2 & 51.6 \\
\hline $\mathrm{ErL}_{\alpha}$ & $119.5\left(\mathrm{ErPO}_{4}\right)$ & 2.1 & 56.9 \\
\hline $\operatorname{TmL}_{\alpha}$ & $122.9\left(\mathrm{TmPO}_{4}\right)$ & 2.5 & 49.2 \\
\hline $\mathrm{YbL}_{\alpha}$ & $128.0\left(\mathrm{YbPO}_{4}\right)$ & 2.6 & 49.2 \\
\hline $\mathrm{LuL}_{\alpha}$ & $131.3\left(\mathrm{LuPO}_{4}\right)$ & 3.4 & 38.6 \\
\hline $\mathrm{PbM}_{\alpha}$ & $72.0\left(\mathrm{PbCO}_{3}\right)$ & 0.6 & 120.0 \\
\hline
\end{tabular}

${ }^{a}$ Average peak and background intensities measured on the primary standards for the analyzed elements along with calculated peak to background ratios. Off-peak positions were based on high-resolution spectral scans of the low to high off-peak regions of each REE element and Pb in each of the REE phosphates. The purpose was to avoid off-peak interferences as much as possible.

Table 4. Typical single analysis and average (replicate) detection limits ${ }^{\mathrm{a}}$

\begin{tabular}{|c|c|c|}
\hline Element & $\begin{array}{l}\text { Detection limit (single point) } \\
(.99 \mathrm{CL})\left(\text { mass fraction } \times 10^{2} \text { in } \mathrm{CePO}_{4}\right)\end{array}$ & $\begin{array}{c}\text { Detection limit (avg. of } 10) \\
(.99 \mathrm{CL})\left(\text { mass fraction } \times 10^{2} \text { in } \mathrm{CePO}_{4}\right)\end{array}$ \\
\hline $\mathrm{ScK}_{\alpha}$ & 0.058 & 0.018 \\
\hline $\mathrm{YL}_{\alpha}$ & 0.103 & 0.024 \\
\hline $\mathrm{LaL}_{\alpha}$ & 0.187 & 0.045 \\
\hline $\mathrm{CeL}_{\alpha}$ & 0.147 (in $\mathrm{GdPO}_{4}$ ) & 0.050 (in $\mathrm{GdPO}_{4}$ ) \\
\hline $\operatorname{PrL}_{\alpha}$ & 0.104 & 0.058 \\
\hline $\mathrm{NdL}_{\alpha}$ & 0.111 & 0.068 \\
\hline $\mathrm{SmL}_{\alpha}$ & 0.103 & 0.042 \\
\hline $\mathrm{EuL}_{\alpha}$ & 0.137 & 0.052 \\
\hline $\mathrm{GdL}_{\alpha}$ & 0.097 & $0.125^{\mathrm{b}}$ \\
\hline $\mathrm{TbL}_{\alpha}$ & 0.139 & 0.046 \\
\hline $\operatorname{DyL}_{\alpha}$ & 0.100 & 0.033 \\
\hline $\mathrm{HoL}_{\alpha}$ & 0.140 & 0.042 \\
\hline $\mathrm{ErL}_{\alpha}$ & 0.097 & 0.042 \\
\hline $\operatorname{TmL}_{\alpha}$ & 0.139 & 0.033 \\
\hline $\mathrm{YbL}_{\alpha}$ & 0.139 & 0.038 \\
\hline $\mathrm{LuL}_{\alpha}$ & 0.142 & 0.043 \\
\hline $\mathrm{PbM}_{\alpha}$ & 0.077 (in $\mathrm{GdPO}_{4}$ ) & 0.045 (in $\mathrm{GdPO}_{4}$ ) \\
\hline
\end{tabular}

${ }^{a}$ Single point analysis detection limits in a matrix of $\mathrm{CePO}_{4}$ at a $99 \%$ confidence level (CL). $\mathrm{A} \mathrm{GdPO} 4$ matrix for $\mathrm{Ce}$ and $\mathrm{Pb}$ was used since $\mathrm{Ce}$ is a major element in $\mathrm{CePO}_{4}$ and $\mathrm{Pb}$ was determined to be inhomogeneous in the $\mathrm{CePO}_{4}$. CL and averaged detection limits for the same matrices at $99 \%$ confidence interval based on the actual measured standard deviation of 10 measurements on each standard are reported.

${ }^{\mathrm{b}} \mathrm{Gd}$ is possibly present as very small, widely dispersed concentrations in the $\mathrm{CePO}_{4}$ which could explain this unusually high calculated detection limit (for example the calculated average detection limit for $\mathrm{GdL} \alpha$ in $\mathrm{DyPO}_{4}$ is 0.07 mass fraction $\times 10^{2}$ ).

\section{Results and Discussion}

\subsection{REE Impurities in the Orthophosphate Standards}

Table 5 shows the trace REE elements measured in each of the orthophosphates at UC Berkeley on the
"Round Robin" mount. One can see that as stated in the original paper by Jarosewich and Boatner [9], the material is generally very pure based on quantitative results from instrumental neutron activation analysis (INAA). The only statistically significant REE contamination anomalies we observed were the presence of approximately $9 \times 10^{-4}$ mass fraction Eu in $\mathrm{GdPO}_{4}$ (Jarosewich 
Table 5. Trace $\mathrm{Pb}$ and REE concentrations in the $\mathrm{REEPO}_{4}$ standards $^{\mathrm{a}}$ (concentrations and uncertainties in mass fraction $\times 10^{2}$ )

\begin{tabular}{|c|c|c|c|c|c|c|c|c|}
\hline USNM \# & $\begin{array}{c}\mathrm{ScPO}_{4} \\
168495\end{array}$ & $\begin{array}{c}\mathrm{YPO}_{4} \\
168499\end{array}$ & $\begin{array}{c}\mathrm{LaPO}_{4} \\
168490\end{array}$ & $\begin{array}{c}\mathrm{CePO}_{4} \\
168484\end{array}$ & $\begin{array}{c}\mathrm{PrPO}_{4} \\
168493\end{array}$ & $\begin{array}{l}\mathrm{NdPO}_{4} \\
168492\end{array}$ & $\begin{array}{l}\mathrm{SmPO}_{4} \\
168494\end{array}$ & $\begin{array}{l}\mathrm{EuPO}_{4} \\
168487\end{array}$ \\
\hline $\mathrm{ScK}_{\alpha}$ & & $.01 \pm .01$ & $.01 \pm .02$ & $.01 \pm .01$ & $.01 \pm .01$ & $.01 \pm .01$ & $.01 \pm .01$ & $.00 \pm .00$ \\
\hline $\mathrm{YL}_{\alpha}$ & $.01 \pm .02$ & & $.01 \pm .01$ & $.01 \pm .01$ & $.00 \pm .01$ & $.02 \pm .03$ & $.01 \pm .02$ & $.00 \pm .00$ \\
\hline $\mathrm{LaL}_{\alpha}$ & $.01 \pm .01$ & $.02 \pm .03$ & & $.00 \pm .00$ & $.03 \pm .05$ & $.02 \pm .04$ & $.03 \pm .03$ & $.01 \pm .01$ \\
\hline $\mathrm{CeL}_{\alpha}$ & $.00 \pm .01$ & $.05 \pm .06$ & $.01 \pm .01$ & & $.03 \pm .04$ & $.03 \pm .04$ & $.03 \pm .04$ & $.02 \pm .02$ \\
\hline $\operatorname{PrL}_{\alpha}$ & $.03 \pm .03$ & $.01 \pm .02$ & $.07 \pm .13^{\mathrm{b}}$ & $.02 \pm .03$ & & $.00 \pm .01$ & $.01 \pm .01$ & $.02 \pm .03$ \\
\hline $\mathrm{NdL}_{\alpha}$ & $.00 \pm .00$ & $.01 \pm .02$ & $.00 \pm .01$ & $.01 \pm .02$ & $.01 \pm .03$ & & $.00 \pm .01$ & $.04 \pm .04$ \\
\hline $\mathrm{SmL}_{\alpha}$ & $.02 \pm .03$ & $.01 \pm .02$ & $.01 \pm .02$ & $.02 \pm .04$ & $.01 \pm .02$ & $.00 \pm .00$ & & $.01 \pm .02$ \\
\hline $\mathrm{EuL}_{\alpha}$ & $.02 \pm .03$ & $.01 \pm .02$ & $.03 \pm .03$ & $.00 \pm .01$ & $.08 \pm .11^{\mathrm{b}}$ & $.03 \pm .05$ & $.03 \pm .02$ & \\
\hline $\operatorname{GdL}_{\alpha}$ & $.02 \pm .03$ & $.02 \pm .03$ & $.03 \pm .06$ & $.04 \pm .05$ & $.01 \pm .03$ & $.02 \pm .03$ & $.00 \pm .00$ & $.02 \pm .05$ \\
\hline $\mathrm{TbL}_{\alpha}$ & $.01 \pm .01$ & $.02 \pm .03$ & $.02 \pm .03$ & $.00 \pm .01$ & $.03 \pm .04$ & $.00 \pm .00$ & $.03 \pm .05$ & $.00 \pm .01$ \\
\hline $\operatorname{DyL}_{\alpha}$ & $.03 \pm .02$ & $.02 \pm .02$ & $.03 \pm .04$ & $.02 \pm .03$ & $.00 \pm .00$ & $.00 \pm .00$ & $.00 \pm .00$ & $.02 \pm .03$ \\
\hline $\mathrm{HoL}_{\alpha}$ & $.01 \pm .02$ & $.01 \pm .02$ & $.02 \pm .03$ & $.02 \pm .03$ & $.00 \pm .00$ & $.00 \pm .01$ & $.00 \pm .00$ & $.00 \pm .00$ \\
\hline $\mathrm{ErL}_{\alpha}$ & $.03 \pm .03$ & $.02 \pm .02$ & $.02 \pm .03$ & $.03 \pm .04$ & $.00 \pm .00$ & $.02 \pm .03$ & $.07 \pm .04$ & $.00 \pm .00$ \\
\hline $\operatorname{TmL}_{\alpha}$ & $.02 \pm .02$ & $.01 \pm .02$ & $.02 \pm .02$ & $.01 \pm .02$ & $.02 \pm .03$ & $.02 \pm .02$ & $.06 \pm .07$ & $.06 \pm .06$ \\
\hline $\mathrm{YbL}_{\alpha}$ & $.00 \pm .00$ & $.03 \pm .04$ & $.02 \pm .03$ & $.04 \pm .05$ & $.04 \pm .04$ & $.03 \pm .03$ & $.02 \pm .03$ & $.02 \pm .03$ \\
\hline $\mathrm{LuL}_{\alpha}$ & $.02 \pm .03$ & $.01 \pm .03$ & $.01 \pm .02$ & $.03 \pm .04$ & $.02 \pm .03$ & $.04 \pm .03$ & $.00 \pm .00$ & $.00 \pm .01$ \\
\hline \multirow[t]{2}{*}{$\mathrm{PbM}_{\alpha}$} & $.00 \pm .00$ & $.01 \pm .01$ & $1.05 \pm .17$ & $1.68 \pm .07$ & $.77 \pm .04$ & $.60 \pm .03$ & $.99 \pm .07$ & $.52 \pm .06$ \\
\hline & $\begin{array}{c}\mathrm{GdPO}_{4} \\
168488\end{array}$ & $\begin{array}{c}\mathrm{TbPO}_{4} \\
168496\end{array}$ & $\begin{array}{c}\mathrm{DyPO}_{4} \\
168485\end{array}$ & $\begin{array}{c}\mathrm{HoPO}_{4} \\
168489\end{array}$ & $\begin{array}{c}\mathrm{ErPO}_{4} \\
168486\end{array}$ & $\begin{array}{l}\mathrm{TmPO}_{4} \\
168497\end{array}$ & $\begin{array}{l}\mathrm{YbPO}_{4} \\
168498\end{array}$ & $\begin{array}{l}\mathrm{LuPO}_{4} \\
168491\end{array}$ \\
\hline $\mathrm{ScK}_{\alpha}$ & $.01 \pm .01$ & $.00 \pm .00$ & $.01 \pm .01$ & $.01 \pm .02$ & $.01 \pm .02$ & $.00 \pm .00$ & $.00 \pm .01$ & $.01 \pm .02$ \\
\hline $\mathrm{YL}_{\alpha}$ & $.01 \pm .02$ & $.01 \pm .02$ & $.07 \pm .05$ & $.02 \pm .03$ & $.02 \pm .03$ & $.01 \pm .01$ & $.04 \pm .03$ & $.03 \pm .03$ \\
\hline $\mathrm{LaL}_{\alpha}$ & $.02 \pm .04$ & $.01 \pm .02$ & $.02 \pm .04$ & $.03 \pm .04$ & $.01 \pm .02$ & $.01 \pm .01$ & $.03 \pm .05$ & $.02 \pm .03$ \\
\hline $\mathrm{CeL}_{\alpha}$ & $.03 \pm .04$ & $.01 \pm .02$ & $.02 \pm .02$ & $.01 \pm .03$ & $.03 \pm .04$ & $.01 \pm .01$ & $.01 \pm .02$ & $.01 \pm .02$ \\
\hline $\operatorname{PrL}_{\alpha}$ & $.01 \pm .02$ & $.01 \pm .03$ & $.01 \pm .02$ & $.02 \pm .03$ & $.02 \pm .04$ & $.01 \pm .03$ & $.02 \pm .05$ & $.01 \pm .02$ \\
\hline $\mathrm{NdL}_{\alpha}$ & $.01 \pm .02$ & $.02 \pm .03$ & $.01 \pm .02$ & $.01 \pm .03$ & $.02 \pm .04$ & $.03 \pm .04$ & $.00 \pm .00$ & $.03 \pm .04$ \\
\hline $\mathrm{SmL}_{\alpha}$ & $.02 \pm .03$ & $.00 \pm .01$ & $.03 \pm .04$ & $.00 \pm .01$ & $.02 \pm .02$ & $.03 \pm .03$ & $.03 \pm .03$ & $.02 \pm .03$ \\
\hline $\mathrm{EuL}_{\alpha}$ & $.09 \pm .06$ & $.03 \pm .03$ & $.00 \pm .01$ & $.00 \pm .01$ & $.01 \pm .03$ & $.01 \pm .01$ & $.01 \pm .02$ & $.01 \pm .01$ \\
\hline $\mathrm{GdL}_{\alpha}$ & & $.01 \pm .02$ & $.01 \pm .02$ & $.00 \pm .01$ & $.00 \pm .00$ & $.02 \pm .02$ & $.02 \pm .03$ & $.01 \pm .01$ \\
\hline $\mathrm{TbL}_{\alpha}$ & $.01 \pm .02$ & & $.02 \pm .03$ & $.01 \pm .01$ & $.00 \pm .00$ & $.02 \pm .03$ & $.02 \pm .03$ & $.01 \pm .03$ \\
\hline $\operatorname{DyL}_{\alpha}$ & $.00 \pm .00$ & $.00 \pm .00$ & & $.00 \pm .00$ & $.02 \pm .04$ & $.00 \pm .00$ & $.06 \pm .05$ & $.01 \pm .03$ \\
\hline $\mathrm{HoL}_{\alpha}$ & $.14 \pm .21^{\mathrm{b}}$ & $.01 \pm .02$ & $.11 \pm .06$ & & $.01 \pm .01$ & $.01 \pm .03$ & $.02 \pm .04$ & $.03 \pm .03$ \\
\hline $\mathrm{ErL}_{\alpha}$ & $.00 \pm .00$ & $.05 \pm .09$ & $.01 \pm .02$ & $.00 \pm .00$ & & $.11 \pm .07$ & $.02 \pm .02$ & $.00 \pm .01$ \\
\hline $\operatorname{TmL}_{\alpha}$ & $.01 \pm .02$ & $.00 \pm .00$ & $.03 \pm .05$ & $.03 \pm .03$ & $.00 \pm .00$ & & $.00 \pm .01$ & $.04 \pm .04$ \\
\hline $\mathrm{YbL}_{\alpha}$ & $.01 \pm .01$ & $.02 \pm .03$ & $.00 \pm .01$ & $.00 \pm .00$ & $.03 \pm .04$ & $.00 \pm .00$ & & $.00 \pm .00$ \\
\hline $\mathrm{LuL}_{\alpha}$ & $.09 \pm .07$ & $.02 \pm .03$ & $.02 \pm .05$ & $.05 \pm .07$ & $.00 \pm .00$ & $.01 \pm .04$ & $.00 \pm .00$ & \\
\hline $\mathrm{PbM}_{\alpha}$ & $.49 \pm .07$ & $.02 \pm .02$ & $.02 \pm .03$ & $.02 \pm .03$ & $.02 \pm .02$ & $.01 \pm .02$ & $.02 \pm .03$ & $.04 \pm .04$ \\
\hline
\end{tabular}

${ }^{a}$ Average trace analyses of REE elements plus $\mathrm{Sc}, \mathrm{Y}$, and $\mathrm{Pb}$ for the USNM REE phosphates in the "Round Robin" mount measured at Berkeley. The quoted uncertainty is the measured one standard deviation value for 10 measurements.

${ }^{\mathrm{b}}$ Large magnitude interference corrections resulting in increasing uncertainty at trace levels. The apparent concentrations and large standard deviations for these three cases could be greatly reduced by using longer acquisition times on the unknown and the standard used for the interference correction.

and Boatner reported $1.9 \times 10^{-5}$ mass fraction $\mathrm{Eu}$ in $\mathrm{GdPO}_{4}$ using INAA), $1.1 \times 10^{-3}$ mass fraction Ho and $7 \times 10^{-4}$ mass fraction $\mathrm{Y}$ in the $\mathrm{DyPO}_{4}($ Jarosewich and Boatner reported $2.47 \times 10^{-3} \mathrm{Ho}$ in $\mathrm{DyPO}_{4}$ using INAA, $\mathrm{Y}$ was not analyzed by INAA), and approximately $1.1 \times 10^{-3}$ mass fraction $\mathrm{Er}$ in the $\mathrm{TmPO}_{4}(\mathrm{Er}$ was not analyzed by Jarosewich and Boatner with INAA). It is difficult to obtain commercially available REE oxide materials that are completely free of other REE impurities due to the nature of the starting materials (REE-rich phosphate and carbonate minerals) that must be processed to extract individual REEs. The apparent concentration of $0.0009 \pm 0.0007$ mass fraction $\mathrm{Lu}$ in $\mathrm{GdPO}_{4}$ is possibly due to an interference of $\mathrm{Gd} \mathrm{L}_{\gamma 1}$ at $1.5928 \AA$ and the $0.0006 \pm 0.0005$ mass fraction Dy in $\mathrm{YbPO}_{4}$ is possibly due to an interference of $\mathrm{Yb} \mathrm{Ll}$ at $1.8946 \AA$ and finally the $0.0004 \pm 0.0003$ mass fraction $\mathrm{Yb}$ in $\mathrm{YPO}_{4}$ is possibly due to an interference of $\mathrm{Y} \mathrm{K} \alpha_{1}$ (II) at $1.658 \AA$. No other interferences could be invoked to explain the other apparent REE concentrations shown in bold in the table. 


\subsection{Pb Impurities in the Orthophosphate Standards}

The results for $\mathrm{Pb}$ in the last row of Table 5 reveal that $\mathrm{Pb}$ is present from almost 0.02 mass fraction down to about 0.005 mass fraction element in seven of the REE orthophosphates in the "Round Robin" mount (in order of decreasing concentration: $\mathrm{CePO}_{4}, \mathrm{LaPO}_{4}, \mathrm{SmPO}_{4}$, $\mathrm{PrPO}_{4}, \mathrm{NdPO}_{4}, \mathrm{EuPO}_{4}$, and $\mathrm{GdPO}_{4}$ ). The remaining REE or thophosphates did not contain $\mathrm{Pb}$ concentrations above the UC Berkeley detection limit of $4.5 \times 10^{-4}$ mass fraction. These measurements consisted of a 10point traverse on a single grain of each REE orthophosphate. Table 6 shows the $\mathrm{Pb}$ homogeneity measurements on the same "Round Robin" mount but performed in College Park with increased sensitivity (longer count times and higher beam currents). The two data sets agree well considering the apparent inhomogeneity of the $\mathrm{Pb}$ contaminated materials.

What is striking is that the $\mathrm{Pb}$ content varies considerably not only within each grain, but even more so from grain to grain, as seen in Table 7 where a number of $\mathrm{Pb}$ measurements (13-16) over the face of the four $\mathrm{CePO}_{4}$ grains in the "Berkeley" mount show tremendous variation between grains from about 0.015 mass fraction to 0.045 mass fraction element.

\subsection{Crystal Structure and Pb Contamination}

Lead is present in significant amounts only in the monoclinic, high-temperature, monazite-structure orthophosphates $\left(\mathrm{LaPO}_{4}\right.$ through $\left.\mathrm{GdPO}_{4}\right)$, and is absent, or nearly so, in the tetragonal, xenotime-structure, compounds $\left(\mathrm{TbPO}_{4}\right.$ through $\mathrm{LuPO}_{4}$ and $\mathrm{ScPO}_{4}$ and $\left.\mathrm{YPO}_{4}\right)$ as can be seen in Fig. 1, where $\mathrm{Pb}$ concentration is plotted as a function of REE atomic number. Boatner and Sales [7] showed that there is a distinct structural change (monoclinic to tetragonal) between $\mathrm{GdPO}_{4}$ and $\mathrm{TbPO}_{4}$ which suggests that the incorporation of $\mathrm{Pb}$ in the monazite structure, and the lack of $\mathrm{Pb}$ incorporation in the xenotime structure orthophosphates, is related to this change in structure. The so-called lanthanide contraction is a continuous decrease in size across the REEs, and may also play a role in this, however, there are no abrupt decreases in the trivalent ionic radii across the REE series (including from $\mathrm{Gd}$ to $\mathrm{Tb}$ ). Our data suggest that the exclusion of the large (e.g., $1.29 \AA$ in eight coordination, [16] divalent lead cation is limited by the space available in the heavy $\mathrm{REEO}_{8}\left(\mathrm{HREEO}_{8}\right)$ polyhedra and that the divalent $\mathrm{Pb}$ ion, or the trivalent HREEs, will not fit easily into the xenotime structure. For the monoclinic orthophosphates, the light $\mathrm{REEO}_{9}$ $\left(\mathrm{LREEO}_{9}\right)$ polyhedra is much larger and can accommodate the divalent $\mathrm{Pb}^{2+}$ ion into the xenotime structure [16].

Table 6. $\mathrm{Pb}$ (mass fraction $\left.\times 10^{2}\right)$ in the "round robin" mount measured in College Park

\begin{tabular}{|c|c|c|c|c|c|c|c|c|}
\hline & $\mathrm{ScPO}_{4}$ & $\mathrm{YPO}_{4}{ }^{\mathrm{b}}$ & $\mathrm{LaPO}_{4}$ & $\mathrm{CePO}_{4}$ & $\mathrm{PrPO}_{4}$ & $\mathrm{NdPO}_{4}$ & $\mathrm{SmPO}_{4}$ & $\overline{\mathrm{EuPO}_{4}}$ \\
\hline \multirow[t]{2}{*}{$\mathrm{PbM}_{\alpha}$} & $.00 \pm .00$ & $.00 \pm .00$ & $.90 \pm .32$ & $1.90 \pm .07$ & $.92 \pm .04$ & $.86 \pm .17$ & $.86 \pm .13$ & $.64 \pm .16$ \\
\hline & $\mathrm{GdPO}_{4}$ & $\mathrm{TbPO}_{4}$ & $\mathrm{DyPO}_{4}$ & $\mathrm{HoPO}_{4}$ & $\mathrm{ErPO}_{4}$ & $\mathrm{TmPO}_{4}$ & $\mathrm{YbPO}_{4}$ & $\mathrm{LuPO}_{4}$ \\
\hline $\mathrm{PbM}_{\alpha}$ & $.39 \pm .16$ & $.00 \pm .00$ & $.00 \pm .00$ & $.00 \pm .00$ & $.00 \pm .00$ & $.00 \pm .00$ & $.00 \pm .00$ & $.00 \pm .00$ \\
\hline
\end{tabular}

\footnotetext{
a Averaged mass fraction $\times 10^{2}$ results of $\mathrm{Pb}$ contamination measurements performed in College Park on the "round robin" mount. The mass fraction detection limit (99\% confidence level) was approximately $140 \times 10^{-6}$. Note that the measured $\mathrm{Pb}$ standard deviations for the uncontaminated materials are significantly smaller than the measurements performed at Berkeley. These results are due to the increased beam current and counting time used at College Park.

${ }^{\mathrm{b}} \mathrm{PbM}_{\beta}$ was used to avoid the $\mathrm{Y}_{1 \gamma 3}$ line.
}

Table 7. $\mathrm{Pb}$ grain to grain variation within the $\mathrm{CePO}_{4}$ material in the "Berkeley" mount ${ }^{\mathrm{a}}$

\begin{tabular}{lcccc}
\hline \hline & Average (concentrations in mass fraction $\left.\times 10^{2}\right)$ & Standard deviation & Minimum & Maximum \\
\hline Grain \#1 & 2.68 & 0.45 & 2.04 & 3.47 \\
Grain \#2 & 2.55 & 0.16 & 2.33 & 2.83 \\
Grain \#3 & 1.54 & 0.04 & 1.48 & 1.59 \\
Grain \#4 & 3.64 & 0.46 & 3.08 & 4.50
\end{tabular}

\footnotetext{
${ }^{\text {a }}$ Average and standard deviations (13-16 points over the face of each grain) of four grains from the "Berkeley" mount mapped in Fig. 1 in elemental mass fraction $\times 10^{2}$. Analytical conditions were $20 \mathrm{keV}, 150 \mathrm{nA}$, and a $10 \mu \mathrm{m}$ diameter beam. Each analysis is the average of 13 to 16 measurements distributed over the face of each grain. Only grain \#3 was relatively homogeneous in $\mathrm{Pb}$.
} 


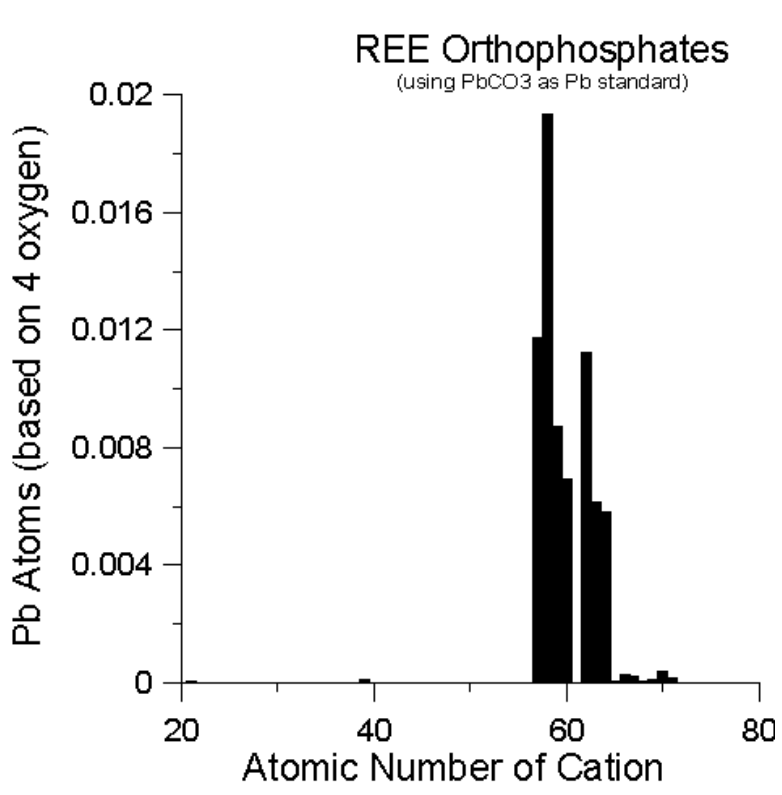

Fig. 1. Plot of $\mathrm{Pb}$ atoms (based on four oxygens) versus atomic number of the REE cation. Element $61(\mathrm{Pm})$ is unstable and the orthophosphate is not available, therefore no measurement is possible for that cation. For all others, only the monoclinic forms for the orthophosphates were observably contaminated by the $\mathrm{Pb}$ flux used to dissolve the starting material prior to crystal growth.

In examining the $\mathrm{REEPO}_{4}$ structures, it is evident that when the REE cation radius contracts beyond a certain point (empirically $1.05 \AA$ ), the REE cation becomes too small to maintain the monoclinic structure type, and the structure distorts to a lower density, lower energy, tetragonal structure type. Once this change from monoclinic to tetragonal symmetry has occurred, the divalent lead cation can no longer fit into this confined $\mathrm{HREEO}_{8}$ polyhedra. The tetragonal orthophosphates are all of the same structure type, with a slight contraction of unit cell volume with increasing atomic number. The same holds true for the monoclinic orthophosphates. There is a dramatic jump in the cell volumes between $\operatorname{Gd}\left(276 \AA^{3}\right)$ and Tb $\left(292 \AA^{3}\right)$ with the phase change.

The tetravalent lead cation with an ionic radius of 0.94 $\AA$ [16], would appear to fit better into the tetragonal xenotime structure orthophosphates with the smaller HREE cations (1.04 $\AA$ to $0.87 \AA,[16])$, but significant $\mathrm{Pb}$ was not observed in those samples. Abraham et al. [8], did find some trivalent $\mathrm{Pb}$ in their EPR experiments, but other valence states of $\mathrm{Pb}$ such as tetravalent lead could have been present since they are not observable by means of EPR spectroscopy [8]. The flux used for crystal growth, $\mathrm{Pb}_{2} \mathrm{P}_{2} \mathrm{O}_{7}$, derived from the decomposition of $\mathrm{PbHPO}_{4}$, contains divalent lead thus, it seems more likely that the $\mathrm{Pb}$ was in the divalent state under the conditions of synthesis.
Characterizing the exact $\mathrm{Pb}$ contamination within a given orthophosphate is problematic because of the degree to which the $\mathrm{Pb}$ concentrations vary, not only within a single grain but also from grain to grain. For this reason it is recommended that each laboratory perform systematic $\mathrm{X}$-ray mapping for $\mathrm{Pb}$ of their "in house" REE orthophosphates grains to determine the actual extent and variation of $\mathrm{Pb}$ contamination in their own mounts. As can be seen in Table 7 (e.g., grain \#3), it may be that the $\mathrm{Pb}$ contamination is homogeneous enough that some portion or another of the material may be suitable for use as a quantitative standard for major element concentrations of the REE in question. Once the $\mathrm{Pb}$ concentration for a homogeneous grain is known and the position noted, the measured $\mathrm{Pb}$ can be proportionally subtracted from the ideal $\mathrm{REEPO}_{4}$ composition and entered into the laboratory's standard compositional database for general use.

Regarding which $\mathrm{REEPO}_{4}$ material should be used for P as an EMPA standard, we suggest that one of the tetragonal orthophosphates should be used to minimize any nonstoichiometry introduced by $\mathrm{Pb}$ impurities.

\section{Conclusions}

Due to their qualities of robustness under the electron beam, resistance to oxidation, and REE purity, the REE orthophosphate standards remain a valuable set of standards for EPMA despite significant $\mathrm{Pb}$ contamination in at least 7 of the 16 samples examined. Of those with measurable $\mathrm{Pb}$ contamination, only the monoclinic $\mathrm{CePO}_{4}$ and possibly the $\mathrm{LaPO}_{4}$ and $\mathrm{SmPO}_{4}$ contain enough $\mathrm{Pb}$ to noticeably affect the stoichiometry for use as a primary standard for major element quantitative analysis (approximately $2 \%$ to $4 \%$ relative differences from their theoretical compositions). None of the tetragonal, xenotime structure orthophosphates $\left(\mathrm{Gd}-\mathrm{LuPO}_{4}\right.$ and $\mathrm{ScPO}_{4}$ and $\mathrm{YPO}_{4}$ ) contain appreciable $\mathrm{Pb}$.

\section{Acknowledgments}

Thanks to Tim Teague at the UC Berkeley Petrographic Laboratory for meticulous sample preparation and to all other researchers who pointed out the presence of $\mathrm{Pb}$ in these materials. 


\section{References}

[1] W. O. Milligan, D. F. Mullica, G. W. Beall, and L. A. Boatner, Structural Investigations of $\mathrm{YPO}_{4}, \mathrm{ScPO}_{4}$, and $\mathrm{LuPO}_{4}$. Inorg. Chim. Acta 60, 39-43 (1982).

[2] W. O. Milligan, D. F. Mullica, G. W. Beall, and L. A. Boatner, Structural Investigations of $\mathrm{ErPO}_{4}, \mathrm{TmPO}_{4}$, and $\mathrm{YbPO}_{4}$. Acta Crystallog. C39, 23-24 (1983).

[3] W. O. Milligan, D. F. Mullica, G. W. Beall, and L. A. Boatner, Structural Investigations of Three Lanthanide Orthophosphates. Inorg. Chim. Acta 70, 133-136 (1983).

[4] W. O. Milligan, D. F. Mullica, G. W. Beall, and L. A. Boatner, Crystal data for lanthanide orthophosphates with zircon-type structure. Inorg. Chim. Acta 77, L23-25 (1983).

[5] D. F. Mullica, D. A. Grossie, and L. A. Boatner, Coordination geometry and structural determinations of $\mathrm{SmPO}_{4}, \mathrm{EuPO}_{4}$, and $\mathrm{GdPO}_{4}$. Inorg. Chim. Acta 109, 105-110 (1985).

[6] D. F. Mullica, D. A. Grossie, and L. A. Boatner, Structural refinements of praseodymium and neodymium orthophosphate. J. Solid State Chem. 58, 71-77 (1985).

[7] L. A. Boatner and B. C. Sales, Monazite in Radioactive Waste Forms for the Future, W. Lutze, R. C. Ewing, eds., Elsevier Science Publishers B.V. (1988) pp. 495-564.

[8] M. M. Abraham, L. A. Boatner, and M. Rappaz, Novel Measurement of Hyperfine Interactions in Solids: $207 \mathrm{~Pb} 3+$ in $\mathrm{YPO}_{4}$ and $\mathrm{LuPO}_{4}$, Phys. Rev. Lett. 45 (10), 839-842 (1980).

[9] E. Jarosewich and L. A. Boatner, Rare-Earth Element Reference Samples for Electron Microprobe Analysis. Geostand. Newslett. Vol XV, 2 (1991).

[10] M. J. Drake and D. F. Weill, New rare earth element standards for electron microprobe analysis. Chem. Geolog. 10, 179-181 (1972).

[11] P. L. Roeder, Electron-Microprobe Analysis of Minerals for Rare-Earth Elements: Use of Calculated Peak Overlap Corrections. Can. Mineralog. 23, 263-271 (1985).

[12] P. L. Roeder, D. MacArthur, X. P. Ma, G. R. Palmer, and A. N. Mariano, Cathodoluminescence and microprobe study of rareearth elements in apatite. Am. Mineralog. 72, 801-811 (1987).

[13] J. J. Donovan, D. A. Snyder, and M. L. Rivers, An improved interference correction for trace element analysis. Microbeam Anal. 2, 23-28 (1993).

[14] V. D. Scott and G. Love, Quantitative Electron-Probe Microanalysis, 2nd Ed., Wiley \& Sons, New York (1983) p. 105.

[15] J. I. Goldstein, D. E. Newbury, P. Echlin, D. C. Joy, C. Fiori, and E. Lifshin, Scanning Electron Microscopy and X-Ray Microanalysis, Plenum, New York (1981) p. 436.

[16] R. D. Shannon, Revised effective ionic radii and systematic studies of interatomic distances in halides and chalcogenides. Acta Crystalog. A32, $751-767$ (1976).

About the authors: John J. Donovan is a research assistant with the University of Oregon, John M. Hanchar is a assistant professor of geochemistry at The George Washington University, Phillip M. Picolli is a research scientist at the University of Maryland, Department of Geology, Marc D. Schrier is as staff scientist at Quantum Dot Corporation, Lynn A. Boatner is a corporate fellow in the Solid State Division at Oak Ridge National Laboratory and Eugene Jarosewich is a research chemist (emeritus) at the Department of Mineral Sciences, the Museum of Natural History, Smithsonian Institution. 